\title{
ON STRUTINSKY'S AVERAGING METHOD
}

\author{
M. BRACK ${ }^{\dagger}$ and $\mathrm{H}$ C. PAULI \\ Institute of Theoretical Physics, University of Basel, Switzerland
}

Received 13 June 1972

(Revised 4 January 1973)

\begin{abstract}
The averaging procedure in Strutınsky's method of shell corrections is formulated for a general type of averaging function. In the case of the harmonic-oscillator potential, the method is proved analytically to give the same results as semiclassical methods. For the infiniterectangular-box potential, an uncertanty in the value of the shell correction $\delta U$ of up to $\approx 0.5 \mathrm{MeV}$ is shown to exist, the semiclassical value lying within these limits. In using realıstic finite-depth potentıals, no restrictions need to be made on the validity of Strutınsky's method, and the uncertainty in the values of $\delta U$ is estumated to be less than $\approx 05 \mathrm{MeV}$.
\end{abstract}

\section{Introduction}

During recent years, Strutınsky's method of shell corrections ${ }^{1-5}$ ) has been successfully used in calculations of the deformation energy of heavy nucle $\left.{ }^{5-13}\right)^{\text {th }}$. Its basic concept, 1 e. the division of the total nuclear binding energy into a smooth liquid-drop energy $E_{\mathrm{LD}}$ and an oscillating shell correction energy $\delta U$, shall not be investigated here; it is discussed in detall in refs. ${ }^{2-5}$ ). We only recall here that the shell correction $\delta U$ is obtained by means of an energy averaging of a single-particle spectrum $\left\{\varepsilon_{t}\right\}$, which has to be the set of eigenvalues of an average nuclear potential, as it is used, e.g., in the shell model.

In the present paper we shall focus on this energy averaging method and compare it to some recently proposed alternative ways of obtaining the shell correction. We shall only consider the first-order shell correction, 1 e the quantity $\delta U=\delta E_{1}$ in the notation of refs. ${ }^{4,5}$ ), thus neglecting higher-order terms. We will also omit pairıngenergy corrections, since they can be calculated separately and added afterwards to the final energy expression.

The shell correction $\delta U$ consists of a proton part and a neutron part Each of them is calculated by taking the difference between the sum of the occupied levels of the nucleus under consideration and its average value, $v i z$.

$$
\delta U_{\tau}=2 \sum_{\imath} \varepsilon_{\imath}^{(\tau)}-2 \int_{-\infty}^{\lambda_{\tau}} E \tilde{g}_{\tau}(E) \mathrm{d} E, \quad \tau=\mathrm{n}, \mathrm{p} .
$$

† Supported by the Swiss National Foundation. Present address: State University of New York at Stony Brook, L.I, New York 11790

${ }^{\dagger \dagger}$ For more extensive reference lists, see the review artıcles refs. ${ }^{5,12.13}$ ). 
For the evaluation of the average level density $\tilde{g}_{\tau}(E)$, Strutınsky proposed a numerical averaging of the single-particle spectrum $\varepsilon_{t}^{(\tau)}$ by Gaussian smoothing functions, taken over a certain energy range $\gamma$. Care has to be taken of the correct value not only of $\tilde{g}_{\tau}(E)$, but also of its derivatives, which makes the so-called curvature corrections necessary. We shall present this formalism in detall in sect. 2, formulating it for a more general type of smoothing function of which the Gaussian is one example. The socalled plateau condition will be discussed, too.

The energy averaging of the single-particle levels, as proposed by Strutinsky, is one possible technical way of determining the average level density entering the definition of the shell correction, eq. (1.1). In fact, the problem of finding an asymptotic expression for the density of eigenstates of a certain potential of given shape has already been considered by Hill and Wheeler ${ }^{14}$ ) for the case of a potential with infinitely deep wells, generalizıng a mathematical result obtained by Weyl ${ }^{15}$ ) in 1911 . Several other authors have taken up this problem again, and different alternative methods have been proposed for obtainıng $\tilde{g}(E)$, most of them based on semiclassical expansions ${ }^{16-23}$ ). It is of some interest to compare their results to Strutınsky's results.

In sect. 3 we shall prove analytically that exactly the same results are obtaned with all methods for the case of the 3-dimensional deformed harmonic-oscillator potential. In the same section, we consider the infinite rectangular potential well. In spite of some small ambiguttes, the method is shown to give results very close to the sem1classical ones in this case, too.

Finally, in sect. 4, some realistıc shell model potentıals are considered and the problem of the influence of unbound states in the case of finite-depth potentials is discussed.

\section{The formalism of Strutinsky's averaging method}

\subsection{EXTRACTION OF A SMOOTH PART OF THE LEVEL DENSITY}

The level density ${ }^{\dagger}$ of a discrete energy spectrum $\varepsilon_{\imath}$ can be written as a sum of $\delta$ functions:

$$
g(E)=\sum_{\imath} \delta\left(E-\varepsilon_{\imath}\right)
$$

The function $g(E)$ varies rapidly with energy, reflecting shell effects due to the nonuniform distribution of the single-particle energies $\varepsilon_{i}$. However, besides its rapidly fluctuating part, $g(E)$ contains also a smooth part $g_{0}(E)$, which describes the average behaviour of the level distribution and which is uniquely determined by the potential to which the efgenvalues $\varepsilon_{\imath}$ belong. Thus, we may write

$$
g(E)=g_{0}(E)+\delta g(E) .
$$

The part $\delta g(E)$ usually oscillates periodically with a typical "wavelength" of the order of $\approx 6-10 \mathrm{MeV}$, which is the mean distance between the main shells occurring in spherical as well as in deformed nuclei ${ }^{2,5,23}$ ).

$\dagger$ For simplicity's sake, we shall throughout this paper consider only one kınd of nucleon. 
It is the goal of the Strutinsky method to extract by means of an energy averaging of the spectrum $\varepsilon_{\imath}$ a smooth quantity $\tilde{g}(E)$ which approximates as well as possible the function $g_{0}(E)$. This is usually achieved ${ }^{1,2}$ ) by smearing out the spectrum $\varepsilon_{\imath}$ with Gaussian functions over an energy range $\gamma$ :

$$
\begin{gathered}
\tilde{g}(E)=\frac{1}{\sqrt{\pi} \gamma} \sum_{\imath}\left\{\mathrm{e}^{-\left(\left(E-\varepsilon_{i}\right) / \gamma\right)^{2}}+\sum_{\mu=1}^{M} a_{2 \mu} \gamma^{2 \mu} \frac{\mathrm{d}^{2 \mu}}{\mathrm{d} E^{2 \mu}} \mathrm{e}^{-\left(\left(E-\varepsilon_{\imath}\right) / \gamma\right)^{2}}\right\}, \\
a_{2 \mu}=(-1)^{\mu} / 2^{2 \mu} \mu ! .
\end{gathered}
$$

By choosing $\gamma$ to be

$$
\gamma \gtrsim \hbar \omega \approx 6-10 \mathrm{MeV},
$$

one obtains a smooth function $\tilde{g}(E)$ which, however, is not necessarily identical to the smooth part $g_{0}(E)$ of the exact level density (2.2). Thus, one chooses the coefficients $a_{2 \mu}$ in the second term of eq. (2.3a), containing the so-called curvature corrections, in such a way that not only $g_{0}(E)$, but also its first $2 M$ derivatives are reproduced correctly. This procedure is described in detall below.

It has been shown by Bassichis et al. ${ }^{24}$ ), that the definition (2.3) of $\tilde{g}(E)$ is equivalent to taking the first $M$ terms of an expansion of the sum of $\delta$-functions (2.1) in terms of Hermite polynomials.

The basic idea of this averaging procedure is not restricted to the use of Gaussian functions only. We shall therefore in the following present the method in a somewhat different way, using a rather general type of averaging functions. The correction coefficients can thereby be determined in a straightforward way.

\section{DETERMINATION OF THE CURVATURE CORRECTIONS}

The energy averaging of the single-particle spectrum $\varepsilon_{1}$ can be represented by means of a folding integral. Defining an integral operator $G_{\text {op }}$ which acts on the quantum level density (2.1),

$$
\boldsymbol{G}_{\mathrm{op}}[g(E)]=\frac{1}{\gamma} \int_{-\infty}^{+\infty} g\left(E^{\prime}\right) f\left(\frac{E-E^{\prime}}{\gamma}\right) \mathrm{d} E^{\prime}
$$

we can write

$$
G(E)=\frac{1}{\gamma} \sum_{\imath} f\left(\frac{E-\varepsilon_{t}}{\gamma}\right)=G_{\mathrm{op}}[g(E)] .
$$

In principle, many different types of averaging functions $f(x)$ can be used. For reasons which become clear in the next section, we will confine ourselves to a class of symmetrical functions specified by the following properties:

(1) The function $f(x)$ is a continuous even function which is positive in $(-\infty,+\infty)$ :

$$
\begin{gathered}
f(x)>0, \quad x \in(-\infty,+\infty), \\
f(-x)=f(x) .
\end{gathered}
$$


(ii) The function $f(x)$ has its maximum value at $x=0$ and falls rapidly off on both sides, going faster to zero than any power of $x^{-1}$ if $x \rightarrow \pm \infty$ :

$$
\begin{gathered}
f^{\prime}(0)=0, \quad f^{\prime \prime}(0)<0, \\
f(x) \leqq f(0), \\
x^{m} f(x) \underset{x \rightarrow \pm \infty}{\rightarrow} 0 \quad(m=0,1,2, \ldots) .
\end{gathered}
$$

(iii) All derıvatıves of $f(x)$ exıst and go to zero as $x \rightarrow \pm \infty$.

$$
\frac{\mathrm{d}^{n}}{\mathrm{~d} x^{n}} f(x) \underset{x \rightarrow \pm \infty}{\rightarrow} 0 \quad(n=1,2,3, \ldots) .
$$

From the properties $(2.7 \mathrm{a}-\mathrm{e})$ follows that all even moments $c_{2 m}$ exist and are finite:

$$
c_{2 m}=\int_{-\infty}^{+\infty} x^{2 m} f(x) \mathrm{d} x<\infty \quad(m=0,1,2, \ldots) .
$$

(The uneven moments $c_{2 m+1}$ are zero by symmetry reasons.) We normalize the function $f(x)$ in such a way that

$$
c_{0}=\int_{-\infty}^{+\infty} f(x) \mathrm{d} x=1 .
$$

To the same extent that $f(x)$ goes to zero for large values of $x$, the averaging operator (2.5) will wipe out the oscillating part $\delta g(E)$ of the exact level density (2.2) as soon as $\gamma \gtrsim \hbar \omega$ [see also ref. ${ }^{17}$ )]. But as stated above, the smooth function $G(E)(2.6)$ is in general not identical to $g_{0}(E)$ in eq. (2.2). However, in the cases where $g_{0}(E)$ is equal to a polynomial in $E$, one can correct $G(E)$ to become equal to $g_{0}(E)$, using the curvature corrections. In order to derive those, we cast $G_{\mathrm{op}}(2.5)$ in the form of a series of differential operators. Inserting a Taylor expansion of $g\left(E^{\prime}\right)$ around $E$ into eq. (2.5) and integrating over $E^{\prime}$, we find

with

$$
G_{\mathrm{op}} \equiv \sum_{\mu=0}^{\infty} b_{2 \mu} \gamma^{2 \mu} \mathrm{d}^{2 \mu} j \mathrm{~d} E^{2 \mu}
$$

$$
b_{2 \mu}=c_{2 \mu} /(2 \mu) !
$$

where $c_{2 \mu}$ are the moments (2.8) of the averaging function. The inverse operator of (2.9), defined by

$$
\boldsymbol{G}_{\mathrm{op}}^{-1}[G(E)] \equiv g(E)
$$

can also be written in the same form:

$$
\boldsymbol{G}_{\mathrm{op}}^{-1}=\sum_{\mu=0}^{\infty} a_{2 \mu} \gamma^{2 \mu} \mathrm{d}^{2 \mu} / \mathrm{d} E^{2 \mu}
$$

The coefficients $a_{2 \mu}$ can be constructed easily from the $b_{2 \mu}$ by multiplying the two series (2.9) and (2.11) with each other and puttıng the coefficients of all powers $\mu \geqq 1$ of $\mathrm{d}^{2} / \mathrm{d} E^{2}$ equal to zero. 
In the appendix, some examples of the averaging function $f(x)$ and the corresponding coefficients $a_{2 \mu}$ are given.

The essential point which leads to the definition of the curvature corrections is now the following: If $g_{0}(E)$ is a polynomial of degree $2 M+1$, all derivatives of order $2 M+2$ or more vanish identically in applying $G_{\text {op }}$ on $g_{0}(E)$. Of course, the identity (2.10) then still holds, if only the first $M$ terms of the operator $G_{\text {op }}^{-1}(2.11)$ are used. Thus, applying the first $M$ terms of $G_{\mathrm{op}}^{-1}$ on $G(E)$ will give us back $g_{0}(E)$

For any level density $g(E)$, irrespectively of its smooth part $g_{0}(E)$ being a polynomal or not, we now define the curvature-corrected uniform level density $\tilde{g}(E)$ as

$$
\tilde{g}(E)=\sum_{\mu=0}^{M} a_{2 \mu} \gamma^{2 \mu} \frac{\mathrm{d}^{2 \mu}}{\mathrm{d} E^{2 \mu}} G(E)=\frac{1}{\gamma} \sum_{\iota}\left\{\sum_{\mu=0}^{M} a_{2 \mu} f^{(2 \mu)}\left(\frac{E-\varepsilon_{\ell}}{\gamma}\right)\right\},
$$

where $a_{2 \mu}$ are the expansion coefficients of the operator $G_{\mathrm{op}}^{-1}(2.11)$ Thus, by the very definition (2.12) of $\tilde{g}(E)$, any component of the level density $g_{0}(E)$ which can be described as a $(2 M+1)$ th order polynomial is identically reproduced in this averaging procedure. In the case of the Gaussian function $f(x)=(1 / \sqrt{ } \pi) \exp \left(-x^{2}\right)$, these defin1tions lead exactly to eq. (2 3).

Concerning the properties (27), (2.8) of the averaging function $f(x)$, we make here the following comment. The convergence (2.7e) is not required for all values of $m$. In fact, we can relax the condition (27e) to be valıd only up to a finite value $m=$ $2 N+1$, so that only the first $N$ moments (2.8) exist. Although the operator $\boldsymbol{G}_{\mathrm{op}}$ cannot be written in the closed form (2.9) any more, one can then still extract a part $\boldsymbol{G}_{\mathrm{op}}^{M}$ consisting of the first $M$ terms $(M \leqq N)$ of the series (2.9), the rest of $\boldsymbol{G}_{\mathrm{op}}$ being an averaging integral over the remaining term in a truncated Taylor series. The curvature corrections are then found in exactly the same way by finding the inverse operator of $G_{\mathrm{op}}^{M}$. This is illustrated by an example in appendix D.

A somewhat different generalization of the method, making use of orthogonal polynomials, has recently been described by Bunatian et al. ${ }^{4}$ ).

\subsection{OCCUPATION NUMBER REPRESENTATION OF THE SHELL CORRECTIONS}

In many cases of application, it is convenient to make use of occupation numbers for describing the quantities appearing in the shell correction method [see also refs. $\left.{ }^{4,5}\right)$ ].

The equation for the particle number $N$ can be used to define some averaged occupation numbers $\tilde{n}_{\imath}$ :

$$
N=2 \int_{-\infty}^{\lambda} \tilde{g}(E) \mathrm{d} E=2 \sum_{i} \int_{-\infty}^{\lambda} \tilde{g}_{i}(E) \mathrm{d} E=2 \sum_{i} \tilde{n}_{i},
$$

where

$$
g(E)=\sum_{\imath} \tilde{g}_{\imath}(E)
$$


Inserting eq. (2.12) into (2.13), we obtain explictlly

with

$$
\tilde{n}_{\iota}=\int_{-\infty}^{t_{i}} f(x) \mathrm{d} x+\sum_{\mu=1}^{M} a_{2 \mu} f^{(2 \mu-1)}\left(t_{t}\right),
$$

$$
t_{\iota}=\left(\lambda-\varepsilon_{\imath}\right) / \gamma .
$$

The uniform energy (see eq. (1.1)) is with these definitions

$$
\tilde{U}=2 \int_{-\infty}^{\lambda} E \tilde{g}(E) \mathrm{d} E=2 \sum_{\imath} \varepsilon_{\imath} \tilde{n}_{\imath}+F,
$$

where the term $F$ is explicitly given by

$$
F=2 \gamma^{2} \sum_{i} \int_{-\infty}^{t_{i}} x \tilde{g}_{i}(x) \mathrm{d} x .
$$

Practically, the contribution of the term $F$ to the uniform energy $\tilde{U}$ is zero. This can be shown in the following way. Differentiating eqs. (2.13) and (2.16) with respect to $\gamma$, we find after elimination of the quantity $\partial \lambda / \partial \gamma$,

$$
\frac{\mathrm{d} \tilde{U}}{\mathrm{~d} \gamma}=2 \int_{-\infty}^{\lambda}(E-\lambda) \frac{\partial}{\partial \gamma} \tilde{g}(E) \mathrm{d} E .
$$

From the definition of $\tilde{g}(E)$, eq. (212), we find the 1dentity

$$
\frac{\partial}{\partial \gamma} \tilde{g}(E) \equiv \frac{\mathrm{d}}{\mathrm{d} E}\left[\sum_{i}\left(\frac{E-\varepsilon_{i}}{\gamma}\right) \tilde{g}_{i}(E)\right],
$$

which holds independently of the averaging function $f(x)$. From (2.18) and (2.19) we find after partial integration

$$
\frac{\mathrm{d} \tilde{U}}{\mathrm{~d} \gamma}=2 \gamma \sum_{\imath} \int_{-\infty}^{t_{\imath}} x \tilde{g}_{\imath}(x) \mathrm{d} x \equiv \frac{1}{\gamma} F .
$$

Thus, the unfform energy becomes equal to

$$
\tilde{U}=2 \sum_{\imath} \varepsilon_{\imath} \tilde{n}_{1}+\gamma \mathrm{d} \tilde{U} / \mathrm{d} \gamma .
$$

In this form, we see that the second term vanishes for all values $\gamma_{0}$ of the smearıng parameter, for which

$$
(\mathrm{d} \tilde{U} / \mathrm{d} \gamma)_{\gamma=\gamma_{0}}=0 .
$$

If the function $\tilde{U}(\gamma)$ has a plateau (see below), eq. (2.22) is fulfilled for all points $\gamma_{0}$ of the plateau. In this case, the occupation numbers $\tilde{n}_{t}$ can be said to contain all necessary information for the uniform energy $\tilde{U}$. We can thus write the shell correction $\delta U$ [eq. (1.1)] as

$$
\delta U\left(\gamma_{0}\right)=2 \sum_{\imath} \varepsilon_{\imath} \delta n_{\imath},
$$


where

$$
\delta n_{\imath}=n_{\imath}-\tilde{n}_{\imath}
$$

and $n_{1}$ are the "Hartree-Fock" occupation numbers which are 1 or 0 according as $\varepsilon_{1}$ is occupied or empty

As an illustration, the quantities $\delta n_{\imath}$ are displayed in fig. 1 for an actual case using the spectrum of a Woods-Saxon potential ${ }^{5}$ ) and Gaussian averaging. It is clearly seen, that a relatively narrow region $\approx \pm \gamma$ around the Fermi energy $\lambda$ is cut out of the spectrum. This demonstrates that the shell corrections $\delta U$ are determined only by the single-particle energies near the Fermı energy. Far from 1t, the contributions from the levels $\varepsilon_{\imath}$ are cancelled as $\delta n_{\imath} \rightarrow 0$ for $\left|\varepsilon_{\imath}-\lambda\right| \gg \gamma$

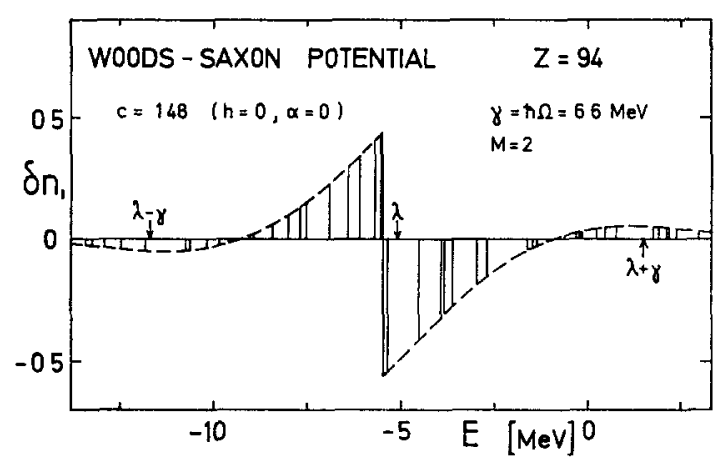

Fig. 1 Proton energy spectrum $\varepsilon_{i}$ of a deformed Woods-Saxon potential For detals of the potential, see ref. ${ }^{5}$ ) The positions of the vertical lines along the energy axis are those of the levels $\varepsilon_{l}$; their lengths are equal to the values of $\delta n_{l}(224)$ The order of curvature corrections is $M=2$

\section{THE PLATEAU CONDITION}

In his original papers ${ }^{1,2}$ ), Strutinsky claimed the shell corrections $\delta U$ to be independent of the parameter $\gamma$ in a region $\hbar \omega \lesssim \gamma \lesssim 2 \hbar \omega$, because the quantity $\gamma$ in itself has no physical meanıng. This so-called plateau condition is well fulfilled for the case of a harmonic-oscillator potential ${ }^{2}$ ) and approximately in a Nilsson model potentıal ${ }^{6}$ ). In more realistic potentials, however, in general no clear plateau is found. The reasons are sketched in the following.

The uniform level density $\tilde{g}(E)$ is introduced so as to represent the smooth part $g_{0}(E)$ of the level density (2.1) which is uniquely defined by the potential. As we have seen above in subsect. $2.2, \tilde{g}(E)$ is identical with $g_{0}(E)$ only if the latter is a pure polynomial in energy. This, in turn, is in general not the case.

The harmonic-oscillator potential makes an exception here. In this special case, $g_{0}(E)$ is known to be a second-order parabola in $E$ [see eq. (3.13) below]. We expect therefore $\tilde{g}(E)$ to be identical with $g_{0}(E)$ as soon as the smearing interval $\gamma$ has reached the value $\hbar \omega$, 1.e. the shell oscillations $\delta g(E)$ in eq. (2.2) are averaged out. In enlarging the interval $\gamma$ even more, no change in $\tilde{g}(E)$ is expected, since the same parab- 
ola holds for $g_{0}(E)$ in any energy region. Thus, an ideal plateau must appear in this case We shall discuss the harmonic-oscillator case in more detall in subsect. 3.1.

The applicability of Strutınsky's method is however not restricted to cases where $g_{0}(E)$ is a pure polynomial. In fact, eqs. $(2.5)$ and $(29)$ demonstrate that $\tilde{g}(E)$ contains the first $2 M$ terms of a Taylor expansion of the level density $g_{0}(E)$ at the energy $E$. Therefore, if $\gamma$ is chosen just large enough to average the oscillations $\delta g(E)$ out, $\tilde{g}(E)$ represents a local approximation of $g_{0}(E)$ by a polynomial of order $2 M$. In order to illustrate this point, let us rewrite the smooth level density $\tilde{g}(E)$, using the defin1toons (2 5) and (2 12)

$$
\begin{gathered}
\tilde{y}(E)=\int_{-\infty}^{+\infty} g_{0}(E+\gamma x) f_{M}(x) \mathrm{d} x, \\
f_{M}(x)=\sum_{\mu=0}^{M} a_{2 \mu} \frac{\mathrm{d}^{2 \mu}}{\mathrm{d} x^{2 \mu}} f(x) .
\end{gathered}
$$

[In (2.25), we have assumed that $\gamma$ is large enough so that the contribution of the part $\delta g(E)$ of $(2.2)$ is practically zero ] We replace now $g_{0}(E+\gamma x)$ in eq $(2.25)$ by the first $2 M+1$ terms of its Taylor expansion around $E$ plus the corresponding remaining term $R_{2 M+1}(E, \gamma x)$

$$
g_{0}(E+\gamma x)=g_{0}(E)+\sum_{\mu=1}^{2 M+1} \frac{(\gamma x)^{\mu}}{\mu !} g_{0}^{(\mu)}(E)+R_{2 M+1}(E, \gamma x)
$$

Then, due to the way in which the curvature corrections have been constructed, $\tilde{g}(E)$ becomes equal to

$$
\tilde{g}(E)=g_{0}(E)+\int_{-\infty}^{+\infty} f_{M}(x) R_{2 M+1}(E, \gamma x)=g_{0}(E)+\Delta g^{M}(E, \gamma) .
$$

Thus, the quantity $\tilde{g}(E)$ is equal to $g_{0}(E)$ up to a small correction $\Delta g^{M}(E, \gamma)$, which is an average of the remainder $R_{2 M+1}$ taken essentially over an interval $E \pm \approx \gamma$. Since this correction is non-vanishing and dependent on $\gamma$ as soon as $g_{0}^{(2 M+2)}(E) \neq 0$, we cannot expect a plateau in this case. However, we can try to make $\Delta g^{M}(E, \gamma)$ as small as possible by looking for its minımum as a function of $\gamma$. Thus, the best approximation of $\tilde{g}_{0}(E)$ might be found by choosing $\gamma$ to be a solution of

$$
\frac{\partial \tilde{g}(E, \gamma)}{\partial \gamma}=\frac{\partial \Delta g^{M}(E, \gamma)}{\partial \gamma}=0
$$

where $\gamma \gtrsim \hbar \omega$. Calculating the uniform energy $\widetilde{U}$ corresponds to approxımating another function of the Fermi energy [due to the integration over $\tilde{g}(E)$, see eq. (2.16)]. Therefore, the best value $\gamma_{0}$ for calculating $\tilde{U}$ should be a solution of

$$
(\partial \tilde{U} / \partial \gamma)_{\gamma=\gamma_{0}}=0,
$$

where we again are interested in solutions $\gamma_{0} \gtrsim \hbar \omega$ only. An upper limit for the parameter $\gamma$ is given first by the finite depth of realistic nuclear potentials, and second 
by the finite number of single-particle levels to be practically used. We shall discuss these limitations in the following sections.

The stationariness condition (2.30) is the infinitesimal equivalent of the usual plateau condition and should be used to determine $\gamma_{0}$ in cases where the latter is not fulfilled. The optımal order $M$ of the curvature correction is reached as soon as the value $\tilde{U}^{(M)}\left(\gamma_{0}^{(M)}\right)$ remains constant when $M$ is increased

$$
\tilde{U}^{(M)}\left(\gamma_{0}^{(M)}\right)=\tilde{U}^{(M+1)}\left(\gamma_{0}^{(M+1)}\right)
$$

The solution of eq. (2.30) is easily found by iteration of $\gamma$ using a starting value $\gamma_{0} \approx \hbar \omega$, where eq. (2.13) has to be fulfilled at each step of the iteration. In iteratıng both quantities $\gamma$ and $\lambda$ simultaneously, sufficient convergence could be reached within 4-8 steps in most practical cases.

\section{Application to some model potentials}

We shall in this section illustrate the shell correction method for two mathematically simple infinite potentials and compare the results to those of some recent semiclassical approaches ${ }^{19,21,23}$ ).

\subsection{ANISOTROPIC 3-DIMENSIONAL HARMONIC OSCILLATOR}

The general form of the 3-dimensional anisotropic harmonic-oscillator potential is

$$
V(x, y, z)=\frac{1}{2} m\left(\omega_{1}^{2} x^{2}+\omega_{2}^{2} y^{2}+\omega_{3}^{2} z^{2}\right) .
$$

The frequencies $\omega_{1}, \omega_{2}, \omega_{3}$ generally differ from each other and may be taken as deformation parameters; in the spherically symmetric case, $\omega_{1}=\omega_{2}=\omega_{3}=\omega_{0}$. Volume conservation is provided for by the condition

$$
\omega_{1} \omega_{2} \omega_{3}=\omega_{0}^{3}=\text { const }
$$

The well-known elgenvalues of the potential (3.1) are

$$
\begin{gathered}
\varepsilon_{n m p}=\varepsilon_{0}+\hbar\left(\omega_{1} n+\omega_{2} m+\omega_{3} p\right)(n, m, p=0,1,2, \ldots), \\
\varepsilon_{0}=\frac{1}{2} \hbar\left(\omega_{1}+\omega_{2}+\omega_{3}\right) .
\end{gathered}
$$

The simple analytical form of the eigenvalues (3.3) allows us to calculate the uniform quantities $\tilde{g}$ and $\tilde{U}$ analytically to a very good approximation. For this purpose, we have to evaluate threefold sums like

$$
\tilde{g}(\lambda)=\frac{1}{\gamma} \sum_{n, m, p=0}^{\infty}\left\{\sum_{\mu=0}^{M} a_{2 \mu} f^{(2 \mu)}\left(\frac{\lambda-\varepsilon_{n m p}}{\gamma}\right)\right\}
$$

[see eq. (2.12)]. Such sums can be calculated using the Euler-MacLaurin formula [see e.g. ref. ${ }^{25}$ )], provided that the averaging parameter $\gamma$ is not considerably smaller than the shell spacing $\hbar \omega_{0}$, i.e.

$$
\gamma \gtrsim \hbar \omega_{0} .
$$


A suitable form of the Euler-MacLaurin formula is the following: If $f(-\infty)=$ $f^{\prime}(-\infty)=.=f^{(2 k+1)}(-\infty)=\ldots=0$, then

$$
\sum_{n=0}^{\infty} f(a-n b)=\frac{1}{b} \int_{-\infty}^{a} f(x) \mathrm{d} x+\frac{1}{2} f(a)+\sum_{k=1}^{\infty} b^{2 k-1} \frac{B_{2 k}}{(2 k) !} f^{(2 k-1)}(a),
$$

where $B_{2 k}$ are the Bernoull numbers. Applying eq. (3.6) to the threefold sum in eq. (3.4), we obtain

$$
\begin{gathered}
\tilde{g}(\lambda)=\frac{1}{\gamma} \sum_{\mu=0}^{M} a_{2 \mu} X_{2 \mu} \\
X_{2 \mu}=\left(\frac{\gamma}{\hbar \omega_{0}}\right)^{3} \int_{-\infty}^{t_{0}} \mathrm{~d} z \int_{-\infty}^{z} \mathrm{~d} y \int_{-\infty}^{y} f^{(2 \mu)}(x) \mathrm{d} x+\frac{\varepsilon_{0} \gamma^{2}}{\left(\hbar \omega_{0}\right)^{3}} \int_{-\infty}^{t_{0}} \mathrm{~d} y \int_{-\infty}^{y} \mathrm{~d} x f^{(2 \mu)}(\lambda) \\
+\left[\frac{\gamma}{12 \hbar}\left(\frac{1}{\omega_{1}}+\frac{1}{\omega_{2}}+\frac{1}{\omega_{3}}\right)+\frac{\varepsilon_{0}^{2} \gamma}{3\left(\hbar \omega_{0}\right)^{3}}\right] \int_{-\infty}^{t_{0}} \mathrm{~d} x f^{(2 \mu)}(x) \\
+\frac{\varepsilon_{0}}{12 \hbar}\left(\frac{1}{\omega_{1}}+\frac{1}{\omega_{2}}+\frac{1}{\omega_{3}}\right) f^{(2 \mu)}\left(t_{0}\right)+\ldots,
\end{gathered}
$$

where

$$
t_{0}=\left(\lambda-\varepsilon_{0}\right) / \gamma
$$

The missing terms in eq. (3.7) all contain higher derivatives $f^{(2 \mu+v)}\left(t_{0}\right)(v=1,3,5, \ldots)$. For the integrals over the function $f(x)$ occurring in eq. (3.7), asymptotic expressions can be inserted if the upper limit $t_{0}$ is much larger than unity (see eqs. (A.2a-c) in the appendix). This sets an upper limit for the averaging parameter,

$$
\gamma \ll \lambda-\varepsilon_{0},
$$

which is not in contradiction to the inequality (3.5), since for medium and heavy nuclei the Fermı energy $\lambda$ lies several units of $\hbar \omega_{0}$ above the lowest energy level $\varepsilon_{0}$. An add1tional upper limit for $\gamma$ is given by the fact that in numerical calculations one is forced to use a finite number of energy levels with a highest level, say, $\varepsilon_{\max }$. Then we must require

$$
\gamma \ll \varepsilon_{\max }-\lambda .
$$

If both conditions (3.9) and (3.10) are fulfilled, we may thus insert the asymptotic expressions (A.2a-c) into eq. (3.7). The result for the uniform level density is for any $M \geqq 1$,

$$
\tilde{g}(\hat{\lambda}) \approx \frac{1}{2\left(\hbar \omega_{0}\right)^{3}} \lambda^{2}-\frac{1}{8 \hbar \omega_{0}} r\left(\omega_{i}\right),
$$

where the deformation-dependent function $r\left(\omega_{1}\right)$ is

$$
r\left(\omega_{i}\right)=\frac{1}{3}\left(\omega_{1}^{2}+\omega_{2}^{2}+\omega_{3}^{2}\right) / \omega_{0}^{2} .
$$

The result (3.11) is independent of $\gamma$ in the region limited by eqs. (3.5), (3.9)and (3.10), and is therefore identical with the plateau value of the curve $\tilde{g}(\lambda, \gamma)$. Numerical com- 
putations of $\tilde{g}(\lambda)$, taken at the plateau, show that the accuracy of eq. (3.11) is better than $\approx 10^{-4}$. This is demonstrated in fig. 2 , where we compare the analytical result (3 11 ) to the numerically calculated $\tilde{g}(\lambda)$ as functions of the Fermi energy. Only at the bottom of the two curves, where eq. (3.9) is not fulfilled, can a slight difference be seen. The same curves are obtained for different deformations of the potential This reflects the fact that the second term in eq. (3.11) is very small compared to the first one and may be neglected in practical cases It is important, however, for the uniform energy $\tilde{U}$.

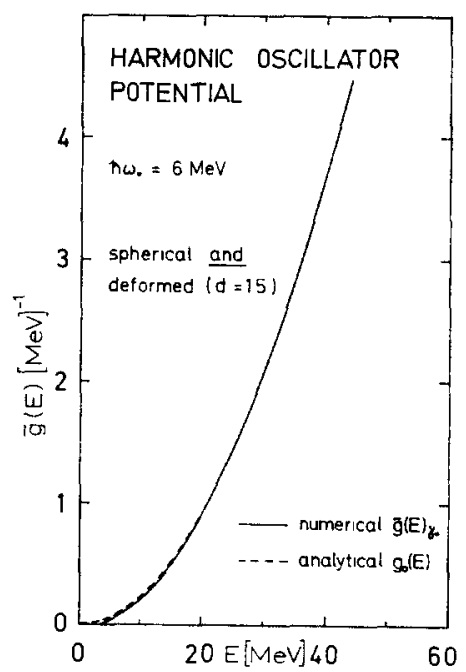

Fig. 2 Uniform level density $\tilde{g}(E)$ for the harmonic-oscillator potential $\left(\hbar \omega_{0}=6 \mathrm{MeV}\right)$, both spherically and axially deformed (ratio of axes $=1.5$ ). The solid line shows the numerical result according to eq. (2 12) using Gaussian averaging. The dashed curve is the analytical result for the platedu value $(311)$ of $\tilde{g}(E)$ which is identical to $g_{0}(E)(3.13)$

It is worth mentioning that the first term in eq. (3.11) is identical to the result of the Thomas-Fermı model applied to the harmonic-oscillator potential. We therefore suppose that eq. (3.11) corresponds to the first two terms of a semiclassical expansion of the local level density. Indeed, the very same result, i.e.

$$
g_{0}(E)=\frac{1}{2\left(h \omega_{0}\right)^{3}} E^{2}-\frac{1}{24 h \omega_{0}} \frac{\omega_{1}^{2}+\omega_{2}^{2}+\omega_{3}^{2}}{\omega_{0}^{2}},
$$

was obtained independently by using a generalized statistical theory ${ }^{19}$ ) and a semiclassical partition function approach ${ }^{21}$ ).

It might be interestıng to note that the result (3.13) can also be obtained by applying the Euler-MacLaurin formula (3.6) directly to the sum of $\delta$-functions (2.1) with the energies $\varepsilon_{n m p}$ (3.3). This calculation is, of course, identical to the limiting case $\gamma \rightarrow 0$ in the above derivation. 
The results for the uniform energy $\tilde{U}$ and the particle number $N$, obtained in the same way as $\tilde{g}(\lambda)$ above, are

$$
\begin{aligned}
& \tilde{U} \approx \frac{1}{4} \frac{1}{\left(\hbar \omega_{0}\right)^{3}} \lambda^{4}-\frac{1}{8 \hbar \omega_{0}} r\left(\omega_{\imath}\right) \lambda^{2}+R\left(\omega_{\imath}\right), \\
& N \approx \frac{1}{3} \frac{1}{\left(\hbar \omega_{0}\right)^{3}} \lambda^{3}-\frac{1}{4 \hbar \omega_{0}} r\left(\omega_{\imath}\right) \lambda,
\end{aligned}
$$

where $r\left(\omega_{\imath}\right)$ is given by eq (3.12) and the third term in eq. (3.14) is explicitly

$$
R\left(\omega_{\imath}\right)=-\hbar \omega_{0}\left[\frac{1}{64} r^{2}\left(\omega_{\imath}\right)+\frac{1}{480} \frac{\omega_{1}^{4}+\omega_{2}^{4}+\omega_{3}^{4}}{3 \omega_{0}^{4}}\right] .
$$

Unlike $\tilde{g}$ and $N$, the uniform energy $\tilde{U}$ (3.14) is only independent of $\gamma$ for $M \geqq 2$. If $M=1$, a term proportional to $\gamma^{4}$ is present, so that $\tilde{U}(\gamma)$ has no plateau at all. This can be seen in fig. 3 , where the shell correction $\delta U$, calculated for the harmonic-

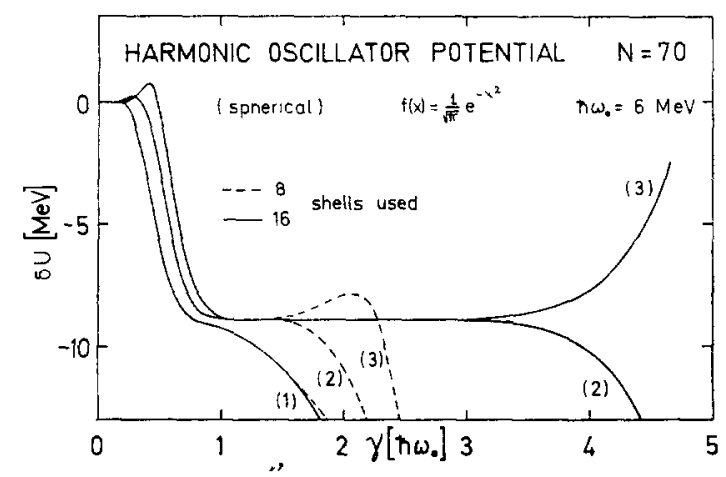

Fig 3. Shell correction $\delta U$ for $N=70$, calculated for the spherical harmonic-oscillator potential, as a function of the smearing parameter $\gamma$. Gaussian averaging has been used; the curvature correction orders $M$ are given in parentheses The number of main oscillator shells used is 16 for the solid lines and 8 for the dashed lines The plateau value is $\delta U=-891 \mathrm{MeV}$

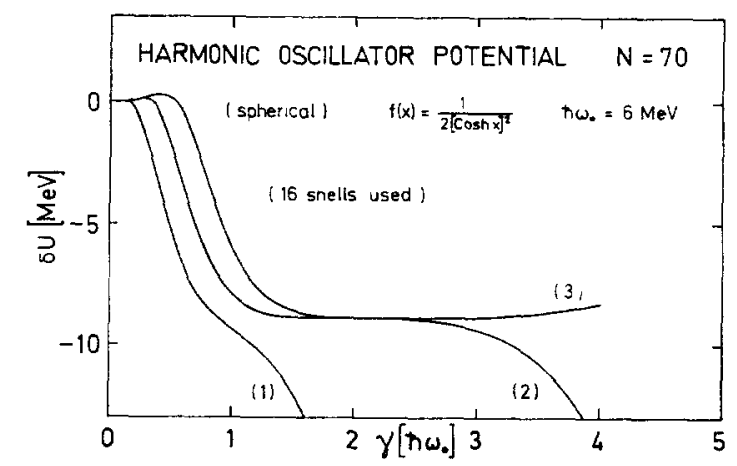

Fig. 4 The same as fig 3, but with the averaging function $f(x)=\frac{1}{2} \cosh ^{-2} x$. The plateau value is $\delta U=-891 \mathrm{MeV}$. 
oscillator potential with $N=70$ using Gaussian averaging, is displayed as a function of $\gamma$ for different orders $M$ of the curvature corrections. The exact numerical results show that the accuracy of eq. (3.14) for the plateau value of $\tilde{U}$ is better than $10^{-5}$. Taking $\hbar \omega_{0}=41(2 N)^{-\frac{1}{3}} \mathrm{MeV}$ [cf. ref. $\left.{ }^{6}\right)$ ], the difference between (3.14) and the exact value of $\tilde{U}$ is less than $\approx 0.03 \mathrm{MeV}$ over the whole range of nucleon numbers $4 \leqq N \leqq 300$ in the spherical case. In fact, the standard deviation of $\approx 0.2 \mathrm{MeV}$ found in the calculations of Bhadurı and Ross ${ }^{21}$ ) turns out to be just of the order of the term $R\left(\omega_{i}\right)$ (3.16) which is missing in their work. The term $R\left(\omega_{\imath}\right)$ can, however, also be obtained in the partition function approach of ref ${ }^{21}$ ), if the energy integral in $\bar{E}$ defined there is interchanged with the integral over the parameter $\beta$ of the inverse Laplace transformation.

The length of the plateau in fig. 3 is clearly dependent on the number of oscillator shells taken into account. In our calculations above, this dependence is determined by the inequality (3.10) which in practice turns out to be more decisive than eq. (39). Thus, the more states above the Fermi energy are used, the longer the plateau will be. Its length is also somewhat dependent on the asymptotic behaviour of the averaging function $f(x)$. This is illustrated in fig. 4 which shows the results obtained with the averaging function $f(x)=\frac{1}{2} \cosh ^{-2} x$. (Its curvature correction coefficients are listed in the appendix.) The curves are very similar to those in fig. 3. As $\cosh ^{-2} x \sim \exp$ $(-2 x)$ for large $x$, the plateau is reached less rapidly than with the Gaussian averaging $\sim \exp \left(-x^{2}\right)$. However, the plateau value of $\delta U$ is exactly the same in both cases. This demonstrates the independence of the method from the special form of the averaging function.

For the understanding of Strutınsky's averaging method it is essential to realize that the length of the plateau is only due to the continuation of the level spectrum above the Fermı energy and has no physical meanıng in itself. The only quantity of physical importance is the plateau value of $\delta U$. Since the uniform energy $\widetilde{U}$ is given by the fourth-order parabola (3.14), the order of the curvature correction can be uniquely determined as $M=2$.

We shall shortly discuss the deformation dependence of the uniform energy $\tilde{U}$ given by eq. (3.14). Eliminatıng the Fermı energy $\lambda$ from eqs. (3 14) and (3.15), we can easıly obtain an expansıon of $\widetilde{U}$ in powers of $(3 N)^{-\frac{2}{3}}$. Retainıng the first two terms, we obtain

$$
\tilde{U}=\frac{1}{4} \hbar \omega_{0}(3 N)^{\frac{4}{2}}\left[1+\frac{1}{2} r\left(\omega_{\imath}\right)(3 N)^{-\frac{3}{3}}\right] .
$$

The next term, which is of zeroth order in $N$, contributes to less than $10^{-4}$, 1.e. to less than $\approx 0.2 \mathrm{MeV}$ in practical cases. Eq. (3.17) for the average part of the single-particle sum, valid for the harmonic-oscillator potential, has recently been obtained by using an expansion appropriate for large nucleon numbers ${ }^{\mathbf{2 3}}$ ).

The first term in eq. (3.17) - the Thomas Fermi term - is proportional to the volume of the nucleus which is kept constant as a function of deformation (see eq. (3.2)). The deformation dependence of $\tilde{U}$ is thus governed by the function $r\left(\omega_{2}\right)$. Strutinsky 
has shown numerically ${ }^{2}$ ) that the deformation-dependent part of $\tilde{U}$ is approximately proportional to the surface area in an axially symmetric harmonic-oscillator potential. (Strictly speaking, the harmonic-oscillator potential defines no clear surface region of the nucleus. The expressions "surface" and "volume" shall thus here only be understood with respect to the deformation dependence of the corresponding terms.) Using our result for $r\left(\omega_{\imath}\right)(3.12)$ in the axially symmetric case, it is easy to confirm the approximate proportionality of this term to the surface of a two-axial ellipsold at moderate deformations.

\section{INFINITE RECTANGULAR BOX IN THREE DIMENSIONS}

We consider a 3-dimensional rectangular box with sides $a, b$ and $c$. Its volume is kept constant by the relation

$$
V=a b c=L^{3} .
$$

The potential is zero inside and infinite outside the box. The eigenvalues of this potential are well known.

$$
\begin{gathered}
\varepsilon_{n m p}=E_{0}\left(\frac{L^{2}}{a^{2}} n^{2}+\frac{L^{2}}{b^{2}} m^{2}+\frac{L^{2}}{c^{2}} p^{2}\right) \quad(n, m, p=1,2,3, \ldots), \\
E_{0}=\frac{\pi^{2} \hbar^{2}}{2 m L^{2}} \approx 51.5(2 N)^{-\frac{3}{3}} \mathrm{MeV},
\end{gathered}
$$

where we have put $L^{3}=\frac{4}{3} \pi r_{0}^{3} A, A=2 N$ and $r_{0}=1.24 \mathrm{fm}$. Due to the quadratic dependence of the $\varepsilon_{n m p}$ on the quantum numbers, it does not seem possible to calculate the Strutinsky averaged quantities $\tilde{g}$ and $\tilde{U}$ analytically in a simple way. It is however still possible to compare these quantities numerically to the results obtained with other methods [cf. also ref. $\left.{ }^{21}\right)$ ].

The average part $g_{0}(E)$ of the density of eigenvalues in a rectangular box has been derived with different semiclassical methods ${ }^{14,20,21}$ ):

$$
g_{0}(E)=\frac{1}{4} \pi E_{0}^{-\frac{3}{2}} \frac{V}{L^{3}} \sqrt{E}-\frac{1}{8} \pi E_{0}^{-1} \frac{S}{2 L^{2}}+\frac{1}{8} E_{0}^{-\frac{1}{2}} \frac{C}{L} \frac{1}{\sqrt{ } E},
$$

where $V$ is the volume (3.18) and $S$ the surface

$$
S=2(a b+a c+b c)
$$

of the box, the quantity $C$ in the "curvature term" of eq. (3.21) is

$$
C=a+b+c .
$$

Since $g_{0}(E)$ (eq. (3.21)) is not a simple polynomial in energy, we have to make use of the stationariness condition (230) in order to find the correct value of the averaging parameter $\gamma$ in each case. Fig. 5 shows some curves $\delta U(\gamma)$, calculated for three arbitrary nucleon numbers. It is seen that the curves in some cases exhibit several stationary points $\gamma_{0}$, which lead to slight ambiguities in the value of $\delta U$. The indeter- 
minacy of $\delta U$ is however not large in these cases, except for low nucleon numbers $(N \lesssim 60)$ where in the worst cases $1 \mathrm{t}$ approaches $\pm \approx 0.5 E_{0}$.

It is very likely that the oscillations of $\delta U(\gamma)$ for values of $\gamma$ larger than $\approx 10 E_{0}$ are due to the fact that the short-range oscillations $\delta g(E)$ in the level density of the spectrum (3.21) are very irregular and do not have a constant "wavelength" (corresponding to $\hbar \omega_{0}$ in the harmonic oscillator). As a consequence, the contribution of $\delta g(E)$ to the averaged quantities $\tilde{g}(E)$ and $\tilde{U}$ is not negligible - an assumption we made in discussıng the stationarıness condition (2.30) in subsect. 2.4. In more realistic potentials, where a main shell spacing $\hbar \omega$ is usually quite well defined, we therefore do not expect serious ambiguities of this kind.

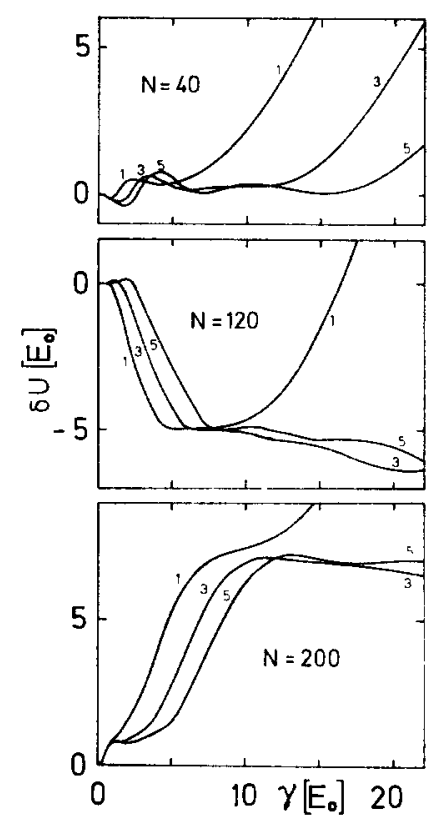

Fig 5 Shell corrections $\delta U$ for the infinite-cubic-box potential as functions of the averaging parameter All energies are in units of $E_{0}$ [see eq. (3.20)] The numbers indicated are the orders $M$ of the curvature correction. (Gaussian averaging used.)

In order to compare the results for $\tilde{U}$ with the semiclassical results, we define the corresponding smooth energy $U_{\mathrm{SCL}}$ [cf. ref. $\left.\left.{ }^{21}\right)\right]$ :

$$
U_{\text {SCL }}=2 \int_{0}^{\lambda_{\mathrm{SCL}}} E g_{0}(E) \mathrm{d} E,
$$

where the Fermı energy $\lambda_{\mathrm{SCL}}$ is found ${ }^{\dagger}$ from

$$
N=2 \int_{0}^{\lambda_{\mathrm{SCL}}} g_{0}(E) \mathrm{d} E .
$$

+ In this equation ( 325$)$, an additional term $-\frac{1}{8} \delta(E)$ has to be added to $g_{0}(E)$, see ref ${ }^{21}$ ). 
Fig. 6 shows the difference $U_{\mathrm{sCL}}-\tilde{U}$, calculated for the case of the cubic box, as a function of the nucleon number $N$. The Strutınsky averaged energy $\tilde{U}$ was calculated using the Gaussian averaging function; the values of the parameter $\gamma$ were given by the equation

$$
\gamma_{0}(N)=(c+00425 N) E_{0},
$$

which was determined empirically to fit the position of the solutions $\gamma_{0}$ of the stationariness condition (2 30) (In the cases of two stationary points $\gamma_{0}$, the value (3.26) fits approximately to therr average.) The constant $c$ in eq. (3.26) depends on the ordet $M$ of the curvature correction

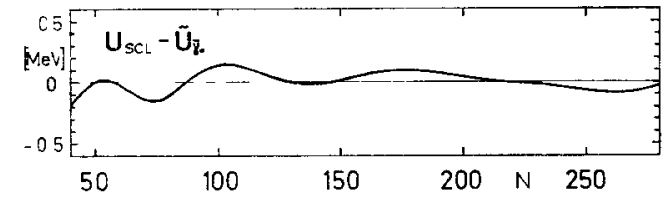

Fig 6. Infinite-cubic-box potential Difference between the semiclassical energy $U_{\text {sCL }}(3.24)$ and the Strutınsky-averaged energy $\tilde{O}$, taken at values $\gamma_{0}(N)=(88+0.0425 N) E_{0}$ (cf eq $\left.(326), M=4\right)$, versus nucleon number $N$. The difference $U_{\mathrm{SCL}}-O$ is given in $\mathrm{MeV}$.

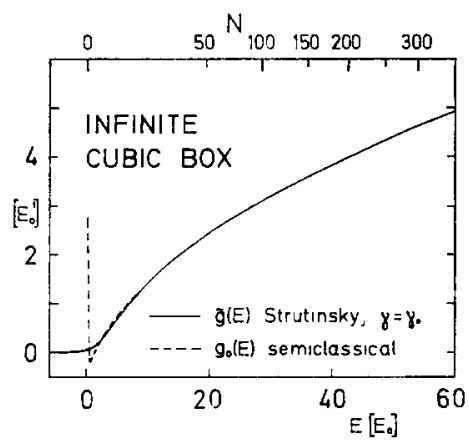

Fig. 7. Level density of the infinite-cubic-box potential as a function of the energy. The corresponding particle numbers are indicated along the upper edge of the figure Solid line the function $\tilde{g}(E)$ taken at the stationary point $\gamma_{0}$ at each energy $E$ (Gaussian averagıng, $M=2$ ) Dashed line: semıclassical level density $g_{0}(E)$, eq $(321)$

Fig. 6 shows that, as an improvement of the conclusions drawn in ref ${ }^{21}$ ), the difference $\left|U_{\mathrm{sCL}}-\tilde{U}\right|$ is less than $\approx 02 \mathrm{MeV}$ for all nucleon numbers $N \gtrsim 40$, if use is made of the stationariness condition (2.30). Qualitatively and quantitatively similar results are obtained for values $2 \leqq M \leqq 4$ of the curvature correction order and for different deformations.

Even including the above ambiguities in the value of $\tilde{U}$, which give uncertainties of up to $\pm \approx 0.5 \mathrm{MeV}$ for $N \gtrsim 60$, we consider the agreement between $\tilde{U}$ and $U_{\text {scL }}$ as being excellent, especially when remembering that their absolute values are of the order of several thousands of $\mathrm{MeV}$. 
The uniform level density $\tilde{g}(E)$ is not very sensitive to the exact value of $\gamma_{0}$, and its agreement with the quantity $g_{0}(E)$ is excellent, as can be seen in fig. 7 . Only around the singular point $E=0$ do the curves differ noticeably.

We want finally to make a comment on the work done by Balian and Bloch ${ }^{20}$ ). These authors used a similar energy averaging - however not including any curvature corrections - with the Lorentzian averaging function

$$
f(x)=\frac{1}{\pi} \frac{1}{1+x^{2}} .
$$

Using Green function techniques, they calculated the smooth part of the level density of an infinite potential well with any given boundary. In the case of the rectangular box, their result is

$$
\begin{aligned}
g_{\gamma}(E) & =\frac{1}{\pi \gamma} \int_{0}^{\infty} \frac{1}{1+\left(\left(E-E^{\prime}\right) / \gamma\right)^{2}} g_{0}\left(E^{\prime}\right) \mathrm{d} E^{\prime} \\
& =\frac{1}{4} \pi E_{0}^{-\frac{3}{2}} \frac{V}{L^{3}} k_{\mathrm{r}}-\frac{1}{8} \pi E_{0}^{-1} \frac{S}{2 L^{2}}\left(1+\frac{2}{\pi} \operatorname{arctg} \frac{k_{1}}{k_{\mathrm{r}}}\right)+\frac{1}{8} E_{0}^{-\frac{1}{2}} \frac{C}{L} \frac{k_{\mathrm{r}}}{k_{\mathrm{r}}^{2}+k_{1}^{2}} .
\end{aligned}
$$

In eq. (3.28), $g_{0}(E)$ is the smooth density $(3.21) ; k_{\mathrm{r}}$ and $k_{1}$ are the real and imaginary parts of the complex number $\sqrt{E+i \gamma}$ :

$$
\begin{aligned}
& k_{\mathrm{r}}=\operatorname{Re} \sqrt{E+i \gamma}=\sqrt{\frac{1}{2}\left(\sqrt{E^{2}+\gamma^{2}+E}\right)}, \\
& k_{1}=\operatorname{Im} \sqrt{E+i \gamma}=\sqrt{\frac{1}{2}\left(\sqrt{E^{2}+\gamma^{2}-E}\right)} .
\end{aligned}
$$

Insertıng the values (3.29) into (3.28), one sees immediately that the density $g_{\gamma}(E)$ becomes close to $g_{0}(E)$ in the limitıng case $\gamma / E \ll 1$. However, in order to average out the shell effects of the single-particle spectrum (3.19), a value of $k_{\imath} \gtrsim 0.5$ is required which corresponds to $\gamma \gtrsim \sqrt{ } E$ (both $\gamma$ and $E$ being measured in units of $E_{0}$ ). Therefore, in applications with nucleon numbers $40 \leqq N \leqq 250$, the parameter $\gamma / E$ has values $\approx \frac{1}{4}$ to $\approx \frac{1}{7}$ (see fig. 7 ) which lead to differences between $g_{\gamma}(E)$ and $g_{0}(E)$ of some $1-2 \%$. These differences may be neglected in studying the level density itself, however, in calculating shell corrections, they turn out to be too large, and curvature corrections to $g_{\mathrm{y}}(E)$ will be indispensable also in the approach of Balian and Bloch. The formalism described above in sect. 2 can be applied, if the generalized type of Lorentzıan curve

$$
f(x)=c_{n} \frac{1}{1+x^{2 n}} \quad(n=2,3,4, \ldots)
$$

is used, which was also considered by Balian and Bloch ${ }^{20}$ ) (see also appendix D).

\section{The shell corrections for realistic potentials}

In sect. 3 we have shown that the Strutınsky averaging method in the cases of two simple infinite potentials leads practically to the same results as the semiclassical meth- 
ods. For the spectra of realistic shell model potentials - especially when the spinorbit term and Coulomb potential are included - no exact analytical method of determining the average part of the level density or the single-particle energy sum seems to exist at present. It is thus a considerable advantage of Strutınsky's method that it can be applied as soon as the single-particle energies are numerically known.

We shall in the following show by means of two examples that the method, using the stationariness condition (2.30), is also able to give unique values of the shell correction for realıstic potentials.

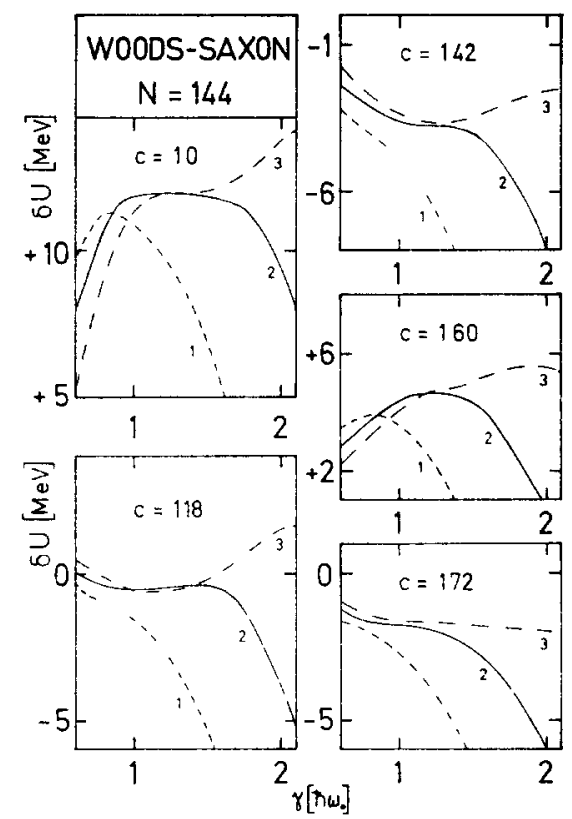

Fig 8. Shell corrections $\delta U$ for the neutron spectrum of a Woods-Saxon potential ${ }^{5}$ ) as functions of $\gamma$, calculated for different deformations Gaussian averaging has been used; the orders $M$ of the curvature corrections are indicated. For the parameters of the potential, see ref. $\left.{ }^{s}\right), c$ is equal to the longer half-axis of the nucleus in units of $R_{0} ; c=1$ is the spherical case $(h=\alpha=0)$. The energy

$$
\hbar \omega_{0}=664 \mathrm{MeV}
$$

\subsection{NILSSON MODEL}

We mention only briefly the calculations done with a modified oscillator potential in the Nilsson model [see e g. ref. ${ }^{6}$ )]. Curves which show $\delta U$ as a function of $\gamma$ for different orders of the curvature correction can be seen in figs. 14 and 15 of ref. ${ }^{6}$ ). (Note that the numbers indicated in these figures equal $2 M$ in our notation.) It is seen that, in general, rather well-pronounced plateaus are obtained. This is not surprising, as the Nilsson potential is infinite and the smooth level density part is not very different from that of the harmonic oscillator. But the plateaus are only well developed if $M \geqq 3$, especially in the deformed cases. This shows that, mainly due to the $\boldsymbol{l}^{2}$ term 
in the potential, there are terms present in the uniform energy, which are of higher order than 4 in the Fermi energy. [The spin-orbit term has been found not to influence much the average level density of a harmonic-oscillator potential, see sect. IV of ref ${ }^{5}$ ). ] However, the plateau value of $\delta U$ is already reached locally in the curves with $M=2$ at a stationary point $\gamma_{0}=(1-1.2) \hbar \omega_{0}$. For higher orders $M$, the plateaus become longer, but their values remain approximately constant. Thus, a fourth-order curvature correction $(M=2)$ seems to be sufficient to give unambiguous values of $\delta U$ for the Nilsson model spectrum, provided that one takes the values of $\delta U$ at the stationary points $\gamma_{0}$

\subsection{FINITE-DEPTH POTENTIALS}

The situation is more critical for finite-depth potentials. In these cases, the average density of the bound states is discontinuous at the upper edge of the potential, being zero in the continuum region. Thus, it is not surprising that no plateau appears in $\delta U(\gamma)$, when the averaging range $\gamma$ is extended several units of $\hbar \omega_{0}$ into the continuum region ${ }^{26}$ ). One possible way to overcome this difficulty is to artificially complete the spectrum outside the region of bound states In the calculations of ref. ${ }^{5}$ ) with a Woods-Saxon potential, the positive eigenvalues, which are obtained by diagonalization of the Hamitonian in a harmonic-oscillator basis, have been used as such art1ficial states. This method has also been used by $\mathrm{Nix}$ and collaborators ${ }^{11,12}$ ), who worked with a folded Yukawa potential [For a discussion of the numerical stability of the unbound states as a function of the cut-off of the basis, see ref. ${ }^{11}$ ).]

In fig. 8, some results are presented which have been calculated with the WoodsSaxon neutron spectrum of ref. ${ }^{5}$ ) [for more detals concerning the parametrization of this potential, see ref. $\left.{ }^{13}\right)$ ]. The curves $\delta U(\gamma)$ are shown for different deformations and curvature correction orders. In most cases, only a local plateau is present. But again, the stationary points $\gamma_{0}$ lie within a physically reasonable region ${ }^{\dagger} \hbar \omega_{0} \lesssim \gamma_{0} \lesssim$ $1.4 \hbar \omega_{0}$ and the values $\delta U\left(\gamma_{0}\right)$ are approximately the same for $M=2$ and $M=3$.

In some cases at large deformations, no stationary point is found below $\gamma \approx 2 \hbar \omega_{0}$. This may be due to a shortcoming of the artificial unbound states used, especially for the protons where the presence of the Coulomb barrier may influence the smooth behaviour of the lowest unbound states. Another reason could be the uncertainty in the value of the shell spacing $\hbar \omega_{0}$ for large deformations, which should rather be determined from the level spectrum itself than by the definition analogous to the quantity $\hbar \omega_{0}$ of the Nilsson model. Nevertheless, the uncertainty in the value of $\delta U$ is not larger than $\approx \pm 0.3 \mathrm{MeV}$, if a mean value of $\gamma_{0} \approx(1.1-1.3) \hbar \omega_{0}$ is used in these few cases.

We should like to emphasize that the only purpose of the artificial unbound states is to continue the spectrum of the bound states in such a way that its smooth behaviour remains unchanged over a larger region above the Fermi energy. In other words, these states may be considered as the hypothetical single-particle states belonging to

\footnotetext{
† In correspondence to the Nilsson model ${ }^{6}$ ), we have defined $\hbar \omega_{0}=41 A^{-\frac{1}{3}}$.
} 
a nucleus with larger nucleon number $A$. In fact, the energy averaging of the level spectrum is equivalent to an averaging over the nucleon number $A$ [cf. refs. ${ }^{2,4,5}$ )]. The unbound states thus extrapolate the average behaviour of the nuclel to those with larger $A$ and have in this sense nothing to do with continuum states. Yet, the resonances caused by the potential in the continuum region may be used to locate the positions of the hypothetical single-particle states belonging to larger nuclel, as has been demonstrated by the recent calculations done by Ross and Bhaduri ${ }^{27}$ ). However, we argue that only little accuracy is gained in evaluating the resonances - which is computationally quite a cumbersome procedure (especially for deformed nuclei)and that the approximate method mentioned above does as well in practical applications.

The use of unbound states or resonances must be considered as merely being a technical trick to find the correct local behaviour of the average level density near the edge of the potential. No other physical influence of the continuum is to be taken into account in Strutınsky's averaging method. In fact, a modıfied prescription has recently been proposed ${ }^{28}$ ) which exclusively takes the bound states into account also in finite-depth potentials.

\section{Summary and conclusions}

The averaging procedure in Strutınsky's shell correction method has been invest1gated. We have shown how the curvature corrections can be found for a general type of averaging function. We emphasize here, that these corrections form an essential part of the method and may by no means be omitted in calculating the uniform ener-

gy $\tilde{U}$. Their contribution to $\tilde{U}$ is usually up to ten times larger than typical values of the shell correction $\delta U$. Thus, comparisons of the Strutınsky averaging with the smoothing due to temperature in statistical calculations should be done very carefully, since in that smoothing no equivalent to the curvature corrections exists [see also refs. $\left.{ }^{4,29}\right)$ ].

We have shown that for two infinite potentials, the Strutinsky averaging leads to the same results for the smooth parts of the level density and the single-particle energy sum as a semiclassical expansion of these quantities. The first three terms of this expansion [see also ref. ${ }^{20}$ )] are proportional to the volume, the surface, and the curvature of the nucleus, respectively. (In the harmonic-oscillator case there is no surface term.) There are no reasons not to expect the presence of a curvature term in the case of realistic potentials, too. Thus, by reasons of consistency, such a curvature term should also be included in the liquid-drop energy $E_{\mathrm{LD}}$ by which the quantity $\widetilde{U}$ is renormalized in the shell correction theory. Recently, the influence of a curvature term in the droplet model has been studied extensively by Hasse ${ }^{30}$ ).

We have shown that the value of the averaging parameter $\gamma$ should be determined by a stationariness condition for the shell correction. With this, Strutinsky's method proves to be valid also for finite-depth potentials, contrary to the conclusions of Lin [ref. $\left.{ }^{26}\right)$ ]. 
As a result of our investigations, we expect an overall accuracy of $\approx \pm 0.5 \mathrm{MeV}$ of the shell corrections. This number is smaller than the value of the second-order shell corrections ${ }^{5}$ ) which have recently been estımated by Bunatian et al. ${ }^{4}$ ) to be of the order of $1 \mathrm{MeV}$. Therefore we conclude that the averaging procedure is accurate enough for all applications to medium and heavy nucleı.

The authors are very much indebted to Profs V. Strutınsky, A Bohr and B. Mottelson for their criticism and valuable comments. We thank Prof. K. Alder for his interest and support of this work.

\section{Appendix A}

ASYMPTOTIC EXPRESSIONS FOR INTEGRALS OVER THE AVERAGING FUNCTION

In eq. (3.7), repeated integrals over the averaging function $f(x)$ occur. The properties (2.7), (2.8) of $f(x)$ allows us to derive asymptotic expressions for such integrals. In order to simplify the notation, we define functions $f_{\mu}(x)(\mu=0, \pm 1, \pm 2, \ldots)$ by

$$
\begin{aligned}
f_{0}(x) & \equiv f(x), \\
f_{\mu-1}(x) & =\int_{-\infty}^{x} f_{\mu}(t) \mathrm{d} t \quad(\mu \leqq 0), \\
f_{\mu+1}(x) & =\frac{\mathrm{d}}{\mathrm{d} x} f_{\mu}(x) \quad(\mu \geqq 0) .
\end{aligned}
$$

From eq. (2.8) follows immediately

$$
f_{-1}(x)=\int_{-\infty}^{x} f(t) \mathrm{d} t \sim 1 \quad(x \gg 1) .
$$

Simılarly, we find from eq. (2 8) by successıve partıal integration

$$
\begin{aligned}
& f_{-2}(x) \sim x \\
& f_{-3}(x) \sim \frac{1}{2} x^{2}+\frac{1}{2} c_{2} \\
& f_{-4}(x) \sim \frac{1}{6} x^{3}+\frac{1}{2} c_{2} x \quad(x \gg 1) \\
& f_{-5}(x) \sim \frac{1}{24} x^{4}+\frac{1}{4} c_{2} x^{2}+\frac{1}{24} c_{4} \\
& \text { etc., }
\end{aligned}
$$

where the $c_{2 \mu}$ are the moments defined by eq. (2.8). Eqs. (2.7e and f) are, of course, equivalent with

$$
f_{\mu}(x) \sim 0, \quad \mu \geqq 0 . \quad(x \gg 1)
$$

\section{Appendix B}

\section{GAUSSIAN AVERAGING}

Usually, the Strutınsky method is employed with the Gaussian averaging function

$$
f(x)=(1 / \sqrt{ } \pi) \mathrm{e}^{-x^{2}} .
$$


The averaging operator $\boldsymbol{G}_{\mathrm{op}}$ [eq. (2.9)] is in this case

$$
\boldsymbol{G}_{\mathrm{op}}=\sum_{\mu=0}^{\infty} \frac{1}{2^{2 \mu} \mu !} \gamma^{2 \mu} \frac{\mathrm{d}^{2 \mu}}{\mathrm{d} E^{2 \mu}}=\mathrm{e}^{(\mathrm{t} \gamma \mathrm{d} / \mathrm{d} E)^{2}}
$$

The inversed operator is thus immediately found to be:

$$
G_{\text {op }}^{-1}=\mathrm{e}^{-(1 / \mathrm{d} / \mathrm{d} E)^{2}},
$$

from which the coefficients (2.3b) follow. The derivatives of the Gaussian (B.1) are

$$
f^{(\mu)}(x)=f_{\mu}(x)=\frac{(-1)^{\mu}}{\sqrt{ } \pi} \mathrm{e}^{-x^{2}} H_{\mu}(x),
$$

with the Hermite polynomials $H_{\mu}(x)$.

TABLE 1

The first moments $C_{2 m}$ and curvature correction coefficients $a_{2 m}$ of the averaging function $f(x)=\frac{1}{2} \cosh ^{-2} x$

\begin{tabular}{rcl}
\hline $2 m$ & $C_{2 m}$ & $a_{2 m}$ \\
\hline 2 & 082247 & -041123 \\
4 & 2.8411 & $+050734 \times 10^{-1}$ \\
6 & 22.175 & $-029804 \times 10^{-2}$ \\
8 & 313.81 & $+010213 \times 10^{-3}$ \\
10 & 70807 & $-0.22926 \times 10^{-5}$ \\
\hline
\end{tabular}

\section{Appendix C}

\section{OTHER EXAMPLES}

We mention only briefly two other examples For the function

$$
f(x)=(1 / \pi) \cosh ^{-1} x
$$

we obtain

$$
a_{2 \mu}=(-1)^{\mu}\left(\frac{1}{2} \pi\right)^{2 \mu} /(2 \mu) !
$$

Another function which may be of interest was used in fig. 4

$$
f(x)=\frac{1}{2} \cosh ^{-2} x .
$$

Its first integral, which gives the occupation numbers in zeroth-order curvature correction [see eq. (2 14)] is equal to the Fermı function:

$$
f_{-1}(x)=\frac{1}{2}[1+\operatorname{tgh} x]=1 /\left(1+\mathrm{e}^{-2 x}\right) .
$$

The next integral $f_{-2}(x)$, used in calculatıng the uniform energy $\tilde{U}$ [see eqs. (2.16), (2.17)], is

$$
f_{-2}(x)=\frac{1}{2}[x+\ln (2 \cosh x)] .
$$

The moments $c_{2 m}$ [eq. (2.8)] cannot be given analytically in this case; we have calculated them numerically and listed them, as well as the $a_{2 m}$, in table 1 . 


\section{Appendix D}

\section{GENERALIZED LORENTZIAN FUNCTIONS}

Although the generalızed Lorentzian curves

$$
f(x)=\frac{n}{\pi} \sin \left(\frac{1}{2 n} \pi\right) \frac{1}{1+x^{2 n}} \quad(n=2,3, \ldots)
$$

do not fulfil all the conditions stated in subsect. 2.2, they can be used with the curvature correction formalism, if the average level density $g_{0}(E)$ grows less rapidly with energy than $E^{2 n-1}$. The first $n-1$ moments of the function (D.1) are finite and equal to

$$
c_{2 m}=\sin \left(\frac{1}{2 n} \pi\right) / \sin \left(\frac{2 m+1}{2 n} \pi\right) \quad(m=0,1, \ldots, n-1) .
$$

In a similar way as in subsect. 2.2, we can write the averaged level density $G(E)$ (cf. eqs. (2.6), (29) and the discussion at the end of subsect. 2.2) as

$$
\begin{aligned}
\int_{-\infty}^{+\infty} g_{0}(E+\gamma x) f(x) \mathrm{d} x=\sum_{\mu=0}^{M} \frac{c_{2 \mu}}{(2 \mu) !} & \gamma^{2 \mu} \frac{\mathrm{d}^{2 \mu}}{\mathrm{d}^{2 \mu}} g_{0}(E)+\int_{-\infty}^{+\infty} f(x) R_{2 M}(E, \gamma x) \mathrm{d} x \\
& =G_{\mathrm{op}}^{M}\left[g_{0}(E)\right]+\Delta g^{M}(E, \gamma) \quad(M \leqq n-1) .
\end{aligned}
$$

where $\boldsymbol{R}_{\mathbf{2}}(E, \gamma x)$ is the remaining term after truncating the Taylor series of $g_{0}$ $(E+\gamma x)$ around $E$ at the $2 M$ th term. The curvature correction coefficients $a_{2 \mu}$ can thus be determined as in subsect. 2.2 by definıng

$$
\left(G_{\mathrm{op}}^{M}\right)^{-1}=\sum_{\mu=0}^{M} a_{2 \mu} \gamma^{2 \mu} \mathrm{d}^{2 \mu} / \mathrm{d} E^{2 \mu} .
$$

In the case $n=3$, e.g., we obtain $a_{0}=1, a_{2}=-\frac{1}{4}, a_{4}=\frac{1}{48}$. Note that eqs. (A.2a-c) hold for the functions (D.1), too.

\section{References}

1) V. M. Strutinsky, Sov. J Nucl. Phys. 3 (1966) 449, Nucl Phys A95 (1967) 420

2) V M. Strutınsky, Nucl. Phys A122 (1968) 1

3) V M. Kolomietz, B D Konstantınov, V. M Strutınsky and B. L Chworostıanov, Kiev preprint ITF-71-93-R (1971)

4) G. G. Bunatian, V. M. Kolomietz and V. M. Strutınsky, Nucl Phys. A188 (1972) 225

5) M. Brack, J. Damgaard, A. S Jensen, H. C. Paul, V M. Strutınsky and C. Y. Wong, Rev. Mod Phys. 44 (1972) 320

6) S. G. Nılsson, C. F. Tsang, A. Sobıczewsk1, Z. Szymansk1, S. Wycech, C Gustafsson, I L. Lamm, P Moller and B. Nilsson, Nucl Phys. A131 (1969) 1

7) B. L. Andersen, F. Dickmann and K Dietrich, Nucl. Phys A159 (1970) 337

8) P. A Seeger and R. C. Perisho, Los Alamos report LA-3751 (1967)

9) V. V. Pashkevich, Nucl. Phys A169 (1971) 275

10) U Gotz, H C Paul,, K. Alder and K. Junker, Phys Lett 38B (1972) 274

11) M Bolsterl, E O. Fiset, J. R Nix and J. L. Norton, Phys. Rev C5 (1972) 1050

12) J R Nix, Ann. Rev Nucl Scı 22 (1972) 
13) H C Paul,, Basel preprint 1972; Phys. Reports, submitted

14) D. L. Hill and $J$ A. Wheeler, Phys. Rev. 89 (1953) 1102

15) H. Weyl, Nachr Kgl. Ges Wiss. Gottıngen, Math -Phys. Klasse (1911) 110

16) M. L. Gursky, Ph.D. thesis, Vanderbilt University, Nashville, Tenn , 1958

17) C F Tsang, Ph D. thesis, University of Calıfornia, Berkeley, 1961, Lawrence Radiation Laboratory report UCRL-18899

18) M Gaudin and A M. Sajot, Proc 2nd IAEA Symp on physics and chemistry of fission, Vienna 1969 , p. 229

19) A S Tyapin, Sov J. Nucl. Phys 11 (1970) 53, 14 (1972) 50

20) R Balian and C Bloch, Ann. of Phys. 60 (1970) 401, 64 (1971) 271; 69 (1972) 76

21) R K Bhadurı and C. K Ross, Phys. Rev Lett. 27 (1971) 606

22) P Siemens and A Sobiczewski, Phys. Lett 41B (1972) 36

23) A. Bohr and B Mottelson, in Nuclear structure, vol. 2 (Benjamın, New York, to be published)

24) W H Bassichis, A. K. Kerman, C F. Tsang, D R. Tuerpe and L. Wilets, Livermore preprint UCRL-73044 (1971)

25) M Abramowitz and I Stegun, Handbook of mathematıcal functions (Dover, New York, 1965) p 806

26) W F Lin, Phys. Rev C2 (1970) 871

27) C K Ross and R K Bhadurı, Nucl. Phys A188 (1972) 566

28) V M Strutinsky, private communication, 1971 , see also appendix of ref ${ }^{4}$ )

29) L G. Moretto, Phys Lett 38B (1972) 393

30) R W. Hasse, Ann. of Phys. 68 (1972) 377 